\title{
Patología otorrinolaringológica ambulatoria en el Hospital Clínico de la Universidad de Chile durante el año 2008: Reporte de 9.157 pacientes
}

\author{
Otolaryngology outpatient pathology at \\ Clinical Hospital of the University of Chile in 2008: Report of 9.157 patients
}

Camila Seymour $\mathbf{M}^{1}$, Javiera Pardo J², Héctor Bahamonde $\mathrm{S}^{3}$.

\section{RESUMEN}

Introducción: En atención primaria las consultas por patología de nariz, oído y faringe son muy frecuentes, representando el $40 \%$ del total.

Objetivo: Describir la patología ambulatoria atendida en el Policlínico de Otorrinolaringología del Hospital Clínico de la Universidad de Chile (HCUCH) durante el año 2008.

Material y método: Estudio retrospectivo descriptivo de las atenciones en el Policlínico de Otorrinolaringología del HCUCH durante el año 2008. Se analizan datos demográficos, diagnósticos y distribución según localización.

Resultados: Se analizan 9.157 pacientes, con promedio de edad de 34,9 años. El $55 \%$ del total correspondió al sexo femenino. El $62 \%$ del total estuvo en el grupo entre 15 y 64 años. Se observó un aumento de las consultas en primavera y verano, representando un tercio. El 38\% correspondió a patología otológica, 27\% nasosinusal, $10 \%$ faríngea y $10 \%$ laríngea. Predominó la hipoacusia (10\%), rinitis alérgica (10\%), patología adenoamigdalina (7\%), otitis media crónica (6\%) y rinosinutis (5\%).

Discusión: Los diagnósticos y su frecuencia encontrados en nuestro centro concuerdan con la literatura internacional.

Conclusión: El conocimiento sobre las patologías más frecuentes y su distribución etaria permiten organizar más eficazmente las atenciones y recursos de nuestro servicio.

Palabras clave: Patología otorrinolaringológica ambulatoria, epidemiología, consultas otorrinolaringológicas.

\section{ABSTRACT}

Introduction: In primary care the consultations about nouse, ear and pharynx's pathology are very frequent, representing the $40 \%$ of the total.

\footnotetext{
${ }^{1}$ Interna de Medicina, Universidad de Chile.

${ }^{2}$ Médico. Tesista de Programa de Magíster en Ciencias Médicas, mención Neurociencias, Universidad de Chile.

${ }^{3}$ Médico. Servicio de Otorrinolaringología, Hospital Clínico Universidad de Chile.
} 
Aim: Describe the outpatient pathology seen at the polyclinic of the Clinical Hospital of the University of Chile in 2008.

Material and method: Retrospective, descriptive study of the Otolaryngology polyclinic consultations during the 2008's year. Demographic datas, diagnosis and distribution by localization were analyzed.

Results: 9.157 patients were analized, with a mean age of 34,9 years. $55 \%$ was female, and the $62 \%$ of the total was in the group between 15 and 64 years. There was a increase of consultations in spring and summer. The $38 \%$ was otologic pathology, $27 \%$ sinonasal and $10 \%$ pharyngeal. It predominated the hearing loss (10\%), allergic rhinitis (10\%), adenotonsillar pathology (7\%), the chronic ear's disease (6\%) and rhinosinusitis (5\%).

Discussion: The diagnoses and their frequencies founded in our center are consistent with international literature.

Conclusions: The knowledge about the most common diseases and age distribution allows to organize more effectively the attentions and resources of our service.

Keys words: Outpatient otolaryngology pathology, epidemiology, otolaryngology consultations.

\section{INTRODUCCIÓN}

Las consultas ambulatorias de otorrinolaringología constituyen una parte importante de la práctica diaria. En atención primaria las consultas por patología de nariz, oído y faringe representan alrededor del $40 \%$ del total ${ }^{1}$. Esta gran prevalencia incide en una gran cantidad de derivación a nivel secundario de pacientes que resultan con enfermedades agudas de manejo de médico general 0 , por otro lado, de pacientes con enfermedades crónicas en fase de agudización.

En cuanto a los diagnósticos más frecuentes presentados en las consultas ambulatorias, se ha reportado un predominio de la patología ótica seguida por las enfermedades crónicas ${ }^{1,2}$. A nivel nacional existen dos estudios realizados, uno en la década de los 70 en el área sur de la región metropolitana, y otro realizado recientemente el año 2009. En relación al primero describe como principal motivo de consulta la patología otológica, y dentro de los diagnósticos más frecuentes, menciona la otitis media crónica seguida por la patología infecciosa adenoamigdalina y la rinosinusal ${ }^{9}$. En el segundo estudio nacional, realizado recientemente, se obtuvieron resultados similares ${ }^{3}$. El presente estudio sería un tercer análisis nacional de la patología otorrinolaringológica.

\section{OBJETIVO}

El objetivo de este estudio fue describir la patología ambulatoria que consulta al Policlínico de Otorrinolaringología del Hospital Clínico de la Universidad de Chile (HCUCH) durante el año 2008.

\section{MATERIAL Y MÉTODOS}

El presente estudio corresponde a un análisis de tipo retrospectivo descriptivo. A partir de las hojas de atención diaria, se analizaron las consultas realizadas en el Policlínico de Otorrinolaringología del HCUCH durante el año 2008. Se analizaron datos demográficos como edad del paciente, sexo y mes de consulta. Además se analizaron diagnósticos más frecuentes, su distribución según localización anatómica y por grupo etario.

\section{RESULTADOS}

Durante el año 2008 se atendieron 9.157 pacientes. El promedio de edad fue de 34,9 años (rango entre 0 y 98 años). El $55 \%$ correspondía a sexo femenino y $45 \%$ masculino. En cuanto a la distribución etaria, el $21 \%$ eran menores de 15 años, el $62 \%$ 
entre 15 y 64 años y el $17 \%$ mayor de 65 años. La distribución porcentual por edad y sexo se muestra en la Figura 1. Se observó un aumento de las consultas en el periodo de primavera y verano, correspondiendo en conjunto a un tercio de las atenciones totales.

Dentro de las consultas, el 39\% correspondía a patología otológica, seguida por la patología rinosinusal (26\%), laríngea (11\%), faríngea (10\%) y el resto correspondiente a patología miscelánea (Figura 2). Las patologías más frecuentes según localización se observan en la Tabla 1.

Los diagnósticos más frecuentes fueron hipoacusia $(10 \%)$, rinitis alérgica $(10 \%)$, patología crónica de adenoides y amígdalas $(7 \%)$, otitis media crónica (OMC) $(6 \%)$ y rinosinusitis aguda 0 crónica (5\%) (Tabla 2).

En relación a la distribución por grupo etario, se observa que en los menores de 15 años existe predominio de la patología adenoamigdalina correspondiente al $25,3 \%$, seguida por la rinitis $(16 \%)$ y la otitis media con efusión (10\%). En cambio, en adultos entre los 15 y 64 años, la patología más frecuente es la rinitis $(9,9 \%)$, seguida por la OMC $(6,8 \%)$.

En el grupo de los adultos mayores, la consulta más frecuente es por hipoacusia $(21,8 \%)$. En segundo lugar se encontró el síndrome vertiginoso $(8 \%)$ y luego la OMC $(6,3 \%)$. La distribución de las principales patologías según grupo etario se observa en la Tabla 3.

\section{DISCUSIÓN}

En este estudio, de los 9.157 pacientes atendidos, predominó el sexo femenino correspondiente al $55 \%$ del total, lo cual concuerda con estudios

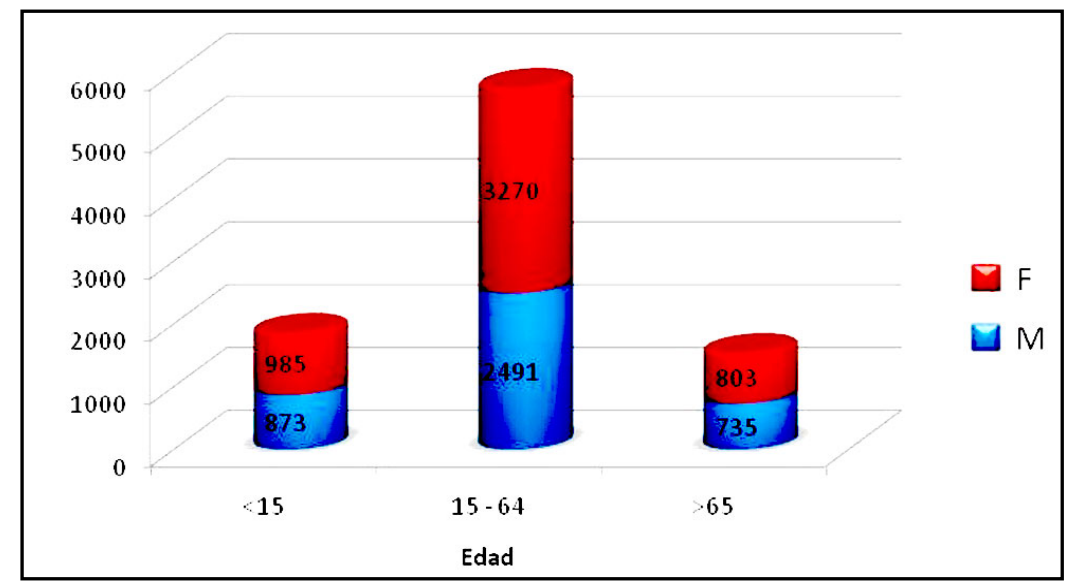

Figura 1. Distribución porcentual por edad y sexo.

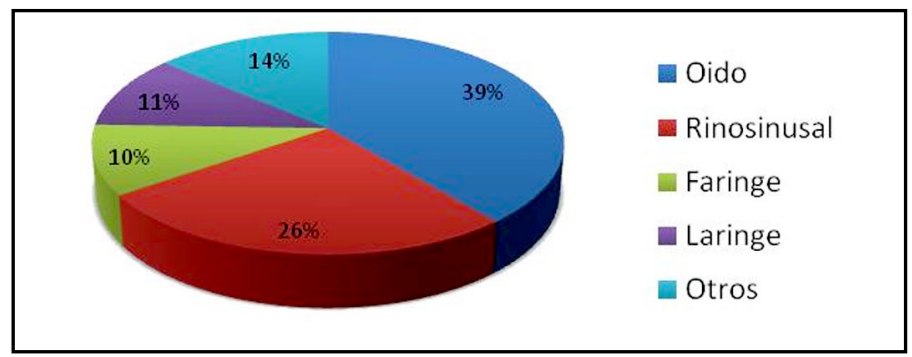

Figura 2. Distribución de los diagnósticos según localización. 
Tabla 1. Patologías más frecuentes según localización

\begin{tabular}{|llr|}
\hline Localización & Diagnóstico & $\%$ relativo \\
\hline \multirow{2}{*}{ Oído } & Otitis media crónica & $16,50 \%$ \\
& Hipoacusia & $15,30 \%$ \\
& Otitis media con efusión & $11,00 \%$ \\
& Síndrome vertiginoso & $10,30 \%$ \\
Rinosinusal & Tapón de cerumen o cuerpo extraño & $9,60 \%$ \\
& Rinitis alérgica & $36,90 \%$ \\
& Dismorfias nasales & $19,10 \%$ \\
& Rinosinusitis aguda y crónica & $14,00 \%$ \\
Faringe & Epistaxis & $6,40 \%$ \\
& Poliposis nasosinusal & $5,90 \%$ \\
& Hiperplasia adenoamigdalina & $65,80 \%$ \\
& Amigdalitis ag, absceso, flegmon & $13,40 \%$ \\
Laringe & Rinofaringitis aguda & $10,20 \%$ \\
& Tonsilolitiasis & $2,60 \%$ \\
& Tumores & $2,00 \%$ \\
& Reflujo faringo-laríngeo & $35,60 \%$ \\
& Disfonía & $27,20 \%$ \\
& Cáncer laríngeo & $9,20 \%$ \\
& Paresia/parálisis cordal & $7,50 \%$ \\
& Laringitis aguda & $6,20 \%$ \\
\hline
\end{tabular}

Tabla 2. Patologías individuales por frecuencia

\begin{tabular}{|lc|}
\hline Diagnóstico & $\%$ del total \\
\hline Rinitis alérgica & $9,80 \%$ \\
Hiperplasia adenoamigdalina & $6,70 \%$ \\
Otitis media crónica & $6,40 \%$ \\
Hipoacusia en estudio & $6,00 \%$ \\
Rinosinusitis aguda y crónica & $5,00 \%$ \\
Dismorfias nasales & $5,00 \%$ \\
Otitis media con efusión & $4,30 \%$ \\
Síndrome vertiginoso & $3,90 \%$ \\
Reflujo faringo-laríngeo & $3,80 \%$ \\
Tapón de cerumen o cuerpo extraño ótico & $3,80 \%$ \\
Otitis externa & $3,20 \%$ \\
Disfonía & $2,90 \%$ \\
Presbiacusia & $2,10 \%$ \\
Epistaxis & $1,70 \%$ \\
\hline
\end{tabular}

previos referentes a consultas ambulatorias de otorrinolaringología ${ }^{1,2,4-6}$. El promedio de edad fue de 34,9 años, lo que se encuentra por debajo a los resultados obtenidos en distintos estudios interna- cionales con promedio entre los 40 y 49 años ${ }^{1,5-7}$, pero similar a los 33 años obtenidos en el estudio nacional de Ruz S y col${ }^{3}$, probablemente explicado por las diferencias en las pirámides poblacionales 
Tabla 3. Distribución de las principales patologías según grupo etario

\begin{tabular}{|clc|}
\hline & Patología & $\begin{array}{c}\% \text { por } \\
\text { grupo etario }\end{array}$ \\
\hline$<15$ años & Patología adenoamigdalina & 25,3 \\
& Rinitis & 16 \\
& OME & 10 \\
& OMC & 3,2 \\
& Rinitis 64 & 9,9 \\
& OMC & 6,8 \\
& Hipoacusia & 4,6 \\
& RFL 64 & 4,4 \\
& Hipoacusia & 21,8 \\
& Síndrome vertiginoso & 8 \\
& OMC & 6,3 \\
& Rinitis & 4,8 \\
& Cáncer de laringe & 3,7 \\
\hline
\end{tabular}

de los países europeos con respecto a nuestro país, el cual posee una población más joven. El grupo comprendido entre los 15 y 64 años correspondieron al $62 \%$ del total, cifra muy distinta al predominio de los menores de 20 años obtenidos en el estudio nacional recién mencionado ${ }^{3}$, pero concordante con los datos internacionales ${ }^{1,2,8,9}$.

La localización más frecuente de los diagnósticos fue la otológica (39\%), lo cual es concordante con la literatura nacional $(42-48 \%)^{3,9} \mathrm{e}$ internacional $(50 \%)^{1,2}$.

En cuanto al diagnóstico más frecuente observado destaca la hipoacusia en primer lugar, seguida cercanamente por la rinitis alérgica, cada una representando el $10 \%$ del total, algo mayor al 7,7\% de frecuencia de rinitis alérgica obtenido en el estudio de Ruz y col. Además en este último estudio predominó la patología crónica de oído, con una prevalencia de $21 \%$ versus el $6,4 \%$ obtenido en este análisis. Aquí cabe destacar que en el estudio mencionado se agrupó las otitis medias crónicas junto a las con efusión en un mismo diagnóstico, sin hacer su distinción, a diferencia del presente análisis. Sin embargo, si agrupamos ambas patologías de todas formas se obtiene una prevalencia menor con respecto al estudio de Ruz y col (11 versus 22\%). Estas diferencias de prevalencia podrían correlacionarse a la disminución de la patología infecciosa actualmente en nuestro país la que ha sido reemplazada, en cierta medida, por enfermedades inflamatorias como rinitis alérgica, además de la muestra de población distinta en la que se hizo el presente estudio la cual probablemente corresponde a un nivel socioeconómico mayor que aquél representado en el trabajo de Ruz.

Según distribución etaria, se observó que en la población infantil predominó la patología adenoamigdalina correspondiente al $25 \%$ del total, seguida por la rinitis y la otitis media con efusión. En adultos la patología más frecuente fue la rinitis $(9,9 \%)$, seguida por la OMC $(6,8 \%)$.

En adultos mayores la consulta más frecuente fue la hipoacusia $(21,8 \%)$. En segundo lugar se encontró el síndrome vertiginoso $(8 \%)$ y luego la OMC $(6,3 \%)$. Sería importante recalcar el aumento de la incidencia de hipoacusia en esta población, comparado con el estudio de Wess y col. Esto probablemente explicado por el envejecimiento progresivo de la población, con una mayor sobrevida actual y por tanto una mayor prevalencia de patologías degenerativas propias de este grupo etario.

\section{CONCLUSION}

La distribución de las patologías según localización encontradas en este estudio, en general concuerdan con la literatura existente, con predominio de la patología otológica y rinosinusal. Sin embargo, se observó una disminución de la incidencia de la otitis media crónica en esta población con respecto a lo publicado por estudios nacionales previos, y una mayor prevalencia de patologías inflamatorias crónicas como rinitis alérgica.

El conocimiento acerca de las patologías más frecuentes y su distribución etaria nos permiten organizar de mejor forma los recursos para optimizar y aumentar la eficacia de nuestro servicio como un centro especialista en otorrinolaringología.

\section{BIBLIOGRAFÍA}

1. García Cantos MD, García Teno M., Rivera García M., Ariza Toledo A. Patología ORL ambulatoria. ORL-DIPS 2005; 32(1): 14-20. 
2. Carbonell R, Pérez F, Jiménez F, Lozano I. Estudio de la demanda asistencial especializada en otorrinolaringología. Rev San Hig Pub 1994; 6: 8(4): 493-502.

3. Ruz S, Breinbahuer $H$, Arancibia M. Análisis epidemiológico de la patología otorrinolaringológica ambulatoria en el Hospital San Juan de Dios. Rev Otorrinolaringol Cir Cabeza Cuello 2009; 69: 227-32.

4. Benito Ji, Morais D, Miyar V, Chamorro J, Martin C. Estudio descriptivo de la asistencia primaria en otorrinolaringología. Acta Otorrinolaringol Esp 1996; 47(1): 55-62.

5. Soto Varela A, Santos Pérez S, Vélez Regueiro mM, Pérez-Carro Ríos A, Labella Caballero T. Estudio epidemiológico descriptivo durante un año de una consulta de otoneurología. An Otorrinolaringol Ibero Am 2000; 27(4): 361-3.

6. Lechuga García R, Frade C, Vaamonde P, Castro C, LABella T. Estudio epidemiológico descriptivo de una consulta de otología durante un año en un hospital de tercer nivel. An Otorrinolaringol Ibero Am 2002; 29 (2): 105-15.
7. García Cano FJ, Sánchez del Hoyo A, Zabaleta López M, Lacosta Nicolas Jl, Martínez Torre I, Preciado LóPEZ JA. Epidemiología descriptiva de las interconsultas hospitalarias realizadas al servicio de ORL durante un año en el complejo hospitalario San Millán-San Pedro. An Otorrinolaringol Ibero Am 2001; 28(5): 487-500.

8. Benito JI, Morais D, Miyar V, Chamorro J, Martin C. Estudio descriptivo de la asistencia primaria en otorrinolaringología. Acta Otorrinolaringol Esp 1996; 47(1): 55-62.

9. Wess I, PeÑA A. Análisis de 19.618 consultas y su proyección hacia la atención y docencia otorrinolaringológica. Rev Otorrinolaring 1976; 36: 5-9.

10. Pino V, Trinidad G, González A et al. Consideraciones sobre las urgencias ORL. Análisis de 30.000 pacientes atendidos en 10 años. Acta Otorrinolaringol Esp 2005; 56: 198-201.

11. Klercker T, Trell E, Lundquist P-G. Essential dataset for ambulatory ear, nose, and throat care in general practice: an aid for quality assessment. Quality in Health Care 1997; 6: 35-9. 